Results: LncMKLN1, dominantly located in nucleus, was up-regulated in lupus patients compared to healthy donors, and could be induced by IFN $\alpha$ and TLR ligands in HRMC. Silencing IncMKLN1 significantly reduced the expression of a group of interferon-inducible genes, including IFIT3, OAS1, CXCL10, etc. We used lose-of-function and gain-of-function strategy through CRSIPR system to confirm that IncMKLN1 positively regulated type I interferon pathway. Furthermore, it was identified the involvement of IncMKLN1 in interferon signalling pathway was through regulating the expression of STAT1, IRF9 and phosphorylation of IRF9 and STAT1 although its mechanism is also needed to investigate. Conclusions: Upregulated IncMKLN1 expression contributed to abnormal activation of interferon pathway of SLE.

Disclosure of Interest: None declared

DOI: 10.1136/annrheumdis-2018-eular.6029

\section{AB0181 ENHANCED THERAPEUTIC EFFICACY OF APOPTOTIC CELL TREATED MESENCHYMAL STEM CELLS IN LUPUS PRONE MRL/LPR MICE}

Z. Zhang, H. Chen, L. Sun. The Affiliated Drum Tower Hospital of Nanjing University Medical School, Nanjing, China

Background: Mesenchymal stem cells (MSCs) exhibit promising therapeutic potential in systemic lupus erythematosus (SLE). Increased apoptotic cells (ACs) were observed in SLE and our previous study showed MSC transplantation reduced $A C$ levels in patients with SLE. Yet the effects of ACs on MSCs are not clear.

Objectives: This study aims to investigate the effects of ACs on MSCs and efficacy of AC treated MSCs (AC-MSCs) in SLE.

Methods: Jurkat T cells were irradiated by ultraviolet ray to induce apoptosis and then co-cultured with umbilical cord derived MSCs. Then, ACs were removed and MSCs were further co-cultured with human peripheral blood mononuclear cells (PBMCs). The inhibition of MSCs on PBMC proliferation was detected by flow cytometry. MSCs and AC-MSCs were infused into lupus prone MRL/lpr mice respectively to compare their therapeutic effects.

Results: The suppression of MSCs on PBMC proliferation was significantly enhanced after co-culture with ACs. In vivo study showed that AC-MSCs significantly increased the survival rate of MRL/Ipr mice and decreased urine protein as early as one week after treatment, while MSCs decreased urine protein eight weeks post infusion. Moreover, AC-MSCs remarkably reduced the number of splenic plasma cells and serum anti-dsDNA levels, whereas MSCs only showed decreased tendency.

Conclusions: ACs enhanced therapeutic effects of MSC transplantation in lupus mice, which provides new insights into MSC modification in the treatment of SLE. Disclosure of Interest: None declared

DOI: 10.1136/annrheumdis-2018-eular.3450

\section{Systemic sclerosis, myositis and related syndromes - etiology, pathogenesis and animal models}

\section{AB0182 MOLECULAR MECHANISMS MEDIATING ANTIOXIDANT EFFECT OF EPIGALLOCATECHIN-3-GALLATE IN EXPERIMENTAL SCLERODERMA MODEL}

A. Kocak ${ }^{1}$, D. Harmancl ${ }^{2}$, M. Birlik ${ }^{3}$, G. Guner ${ }^{4} .{ }^{1}$ Molecular Medicine, Dokuz Eylul University, Institute of Health Sciences; ${ }^{2}$ Molecular Medicine, Dokuz Eylul University, Institute of Health Sciences, Molecular Medicine Dpt, ${ }^{3}$ Dokuz Eylul University, Faculty of Medicine, Rheumatology; ${ }^{4}$ Biochemistry, Izmir University of Economics, Izmir, Turkey

Background: Scleroderma (SSc) is an autoimmune multisystemic connective tissue disease characterised by skin and internal organ fibrosis. ${ }^{1}$ Underlying mechanism is still unclear for SSc. Besides there is no specific treatment for SSc, various treatments may alleviate symptoms and improve the quality of life. Epigallocatechin-3-gallate (EGCG) is a phenol with antioxidant effects in many disease processes. ${ }^{2}$ In this disease, oxidative stress may play a role for pathogenesis $\left(.^{3-}\right.$ ${ }^{4}$ Recent studies showed a relationship between oxidative/antioxidative markers and SSc. ${ }^{5}$

Objectives: The aim of this study was to investigate the antioxidant effects of epigallocatechin-3-gallate in the scleroderma process in experimental mouse model with bleomycin.

Methods: Thirty-two healthy female Balb-c mouse species were used and randomly divided into four groups:control, bleomycin, bleomycin +EGC, EGCG. At the end of the experiment, skin tissues were collected. Sodium dismutase enzyme (SOD) and malondialdehyde (MDA) levels have been analysed for oxidative stress. High performance liquid chromatography (HPLC) was used for MDA measurements. Colorimetric kit was used for SOD analysis. Furthermore, the ratio of phosphorylated p-38/total p-38 protein, and phosphorylated-Akt/total Akt protein and NF-kappa B were measured by western blotting. Immunohistochemistry $(\alpha-S M A)$, histochemistry (masson trichrome-hematoxylin and eosin) studies were also performed on FFPE skin samples.

Results: When the experimental and control groups were compared, the degree of fibrosis in the connective tissue of the dermis areas stained with masson trichrome decreased in the EGCG groups.SOD activity was increased in the EGCG groups compared to the positive control group, and MDA was significantly decreased in the EGCG groups. According to Western blotting results, pp-38 MAPK and NF-KB were found to decrease significantly in the EGCG groups compared with the controls. Parallel to these findings, phosphorylated Akt protein was found to increase in the EGCG groups compared with the control groups.

Conclusions: It has been shown that EGCG can antioxidative effect in scleroderma.

\section{REFERENCES :}

[1] Varga J, Abraham D. Systemic sclerosis: a prototypic multisystem fibrotic disorder. J Clin Invest 2007:117(3):557-67.

[2] White OP, Tribout H, Baron E. Protective mechanisms of green tea polyphenols in skin. Oxid Med Cell Longev 2012;560682.

[3] Herrick AL, Matucci Cerinic M. The emerging problem of oxidative stress and the role of antioxidants in systemic sclerosis. Clin Exp Rheumato 2001;19:4-8

[4] Lundberg AC, Akesson A, Akesson B. Dietary intake and nutritional status in patients with systemic sclerosis. Ann Rheum Dis 1992:51:1143-8.

[5] Kawashiri SY, Ueki Y, Terada K, Yamasaki S, Aoyagi K, Kawakami A. Improvement of plasma endothelin-1 and nitric oxide in patients with systemic sclerosis by bosentan therapy. Rheumatol Int 2014;34:221-5.

Acknowledgements: This research carried out at Dokuz Eylul University Medicine Faculty of Research Laboratory (R-LAB).

Disclosure of Interest: None declared

DOI: 10.1136/annrheumdis-2018-eular.6677

\section{AB0183 VITAMIN D AND VITAMIN D RECEPTOR IN PATIENTS WITH SCLERODERMA SUBTYPES}

A. Kocak ${ }^{1}$, D. Harmanc1 ${ }^{1}$, M. Birlik ${ }^{2}$, G. Guner ${ }^{3} .{ }^{1}$ Molecular Medicine, Dokuz Eylul University, Institute of Health Sciences, Molecular Medicine Dpt, ${ }^{2}$ Dokuz Eylul University, Faculty of Medicine, Rheumatology, ${ }^{3}$ Biochemistry, Izmir University of Economics, Izmir, Turkey

Background: The aim of this study was to compare the expression of Vitamin $D$ receptor (VDR) and vitamin D in scleroderma subtypes.

Objectives: VDR is a member of the nuclear localised hormone receptor family. 1,25- $(\mathrm{OH}) 2 \mathrm{D}$, a form of metabolically active Vitamin $\mathrm{D} 3$, is the ligand of VDR. When VDR and 1,25- $(\mathrm{OH}) 2 \mathrm{D}$ are linked, many genes initiate molecular interaction reactions that will modulate the transcription. ${ }^{1}$ VDR has been shown to be a negative regulator of the TGF- $\beta /$ Smad signalling pathway, which is important in the pathogenesis of scleroderma. ${ }^{2,3}$ Thus, reduced expression of VDR and decreased ligand levels may contribute to hyperactivity of the TGF-beta pathway in SSC and abnormal fibroblast activation. Also, Vitamin D has pleiotropic effects including immunomodulatory and antifibrotic properties in scleroderma pathogenesis.

Methods: 19 SSC patients and 6 healthy controls were included in the study and they were classified according to the 2013 ACR/EULAR criteria. They were applied to Dokuz Eylul University, Faculty of Medicine, Department of Rheumatology-Immunology, between 2015-2017. Rodnan scores were calculated of al scleroderma patients. 11 were of the limited type and 8 were of the diffuse type of scleroderma. Informed consent was obtained from all participants. $1 \mathrm{ml}$ of total blood was collected. Vitamin D levels were determined in serum.

VDR gene expression was determined by quantitative PCR in isolated RNAs from the blood. Changes in mRNA levels were analysed according to the $\triangle \Delta C T$ method and beta-actin was used as the housekeeping gene. Student-t-test was used as a statistic. In addition, Pearson correlation test was used to determine the relationship between Rodnan score and VDR gene expression.

Results: VDR gene expressions in diffuse type scleroderma patients were statistically significantly decreased compared to the control $(p<0.01)$

It was found that VDR gene expression in limited type scleroderma patients did not show any significant difference when compared to control ( $p: 0.16)$.

Also, Vitamin $D$ levels and vitamin $D$ expressions were no correlation in scleroderma subtypes $(\mathrm{p}: 0.2)$

Conclusions: VDR gene expression decreased in patients with diffuse type scleroderma and showed negative correlation with Rodnan score. Further studies are planned to increase the number of samples to obtain more information. 